\title{
Common atherosclerosis genetic risk factors and subclinical atherosclerosis in rheumatoid arthritis: the relevance of disease duration
}

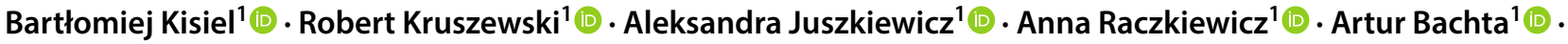

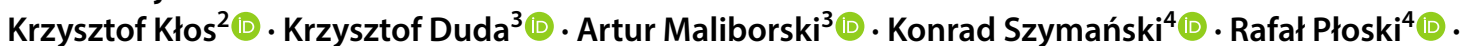

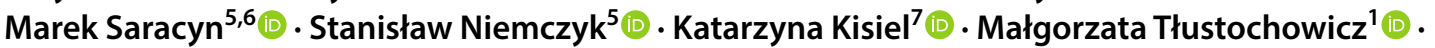 \\ Witold Tłustochowicz ${ }^{1}$
}

Received: 9 August 2018 / Accepted: 21 October 2018 / Published online: 29 October 2018

(c) The Author(s) 2018

\begin{abstract}
Rheumatoid arthritis (RA) is a common systemic autoimmune disease characterized by increased cardiovascular morbidity. Several previous studies assessed associations between common atherosclerotic genetic risk factors and subclinical atherosclerosis (SA) in RA patients, yet most of them gave negative results. We undertook a cross-sectional study to evaluate the association between previously reported SNPs and subclinical atherosclerosis in a cohort of Polish RA patients. 29 SNPs associated with atherosclerosis in general population were genotyped in 289 RA patients: 116 patients with SA (increased carotid intima-media thickness and/or presence of carotid plaque) and 173 patients without SA. To assess the cumulative effect of SNPs we calculated 3 weighted genetic risk scores: $\mathrm{GRS}_{\mathrm{IMT}}, \mathrm{GRS}_{\mathrm{CP}}$ and GRS $\mathrm{CAD}_{\mathrm{D}}$, comprising intima-media thickness-associated SNPs, carotid plaque-associated SNPs and coronary artery disease-associated SNPs, respectively. None of the SNPs showed a significant association with SA. However, we found an association between SA and GRS IMT $_{\text {. }}$ Interestingly, this association was limited to patients with short disease duration $(P=0.00004$ vs. $P>0.5$, for comparison of $\mathrm{GRS}_{\mathrm{IMT}}$ among patients within the 1st quartile of disease duration vs. others, respectively). Patients within the 1st quartile of disease duration were more frequently disease modifying anti-rheumatic drugs (DMARDs)-naïve and less frequently treated with biologics. Our study suggests that in patients with early RA subclinical atherosclerosis may be driven by similar genetic factors as in general population, while in long-lasting disease, the role common genetic risk factors may decrease. Possibly, this effect may be due to the influence of DMARDs.
\end{abstract}

Keywords Atherosclerosis $\cdot$ Rheumatoid arthritis - Intima-media thickness $\cdot$ Disease modifying anti-rheumatic drugs

\section{Introduction}

Rheumatoid arthritis (RA) is a common systemic autoimmune disease characterized by chronic joint inflammation and extra-articular manifestations. It is well known that cardiovascular disease (CVD) morbidity and mortality rates are increased in RA patients as compared to general population

Electronic supplementary material The online version of this article (https://doi.org/10.1007/s00296-018-4186-y) contains supplementary material, which is available to authorized users.

Bartłomiej Kisiel

bartlomiejkisiel@wp.pl

Extended author information available on the last page of the article
[1]. It has been shown that the excess of cardiovascular (CV) risk in RA persists after adjustment for classical $\mathrm{CV}$ risk factors [2], thus RA is considered as an independent $\mathrm{CV}$ risk factor.

Assessment of intima-media thickness in carotid arteries (CIMT) is one of the methods used for detecting subclinical atherosclerosis. A systematic review and meta-analysis by Lorenz et al. showed that CIMT predicts future vascular events in healthy individuals [3]. Tyrrell et al. found in their meta-analysis that CIMT is increased in RA [4].

Coronary artery disease (CAD) is a disease with a strong genetic component. Previous large-scale genetic studies identified nearly 50 loci associated with CAD [5]. However, a study by Conde et al. showed no association between several CAD-associated SNPs and CIMT, which suggests 
different genetic bases for subclinical atherosclerosis and coronary artery disease [6]. Similar results were reported by Zabalza et al.- no association was found between 9 CAD-associated variants and 3 subclinical atherosclerosis measurements (including CIMT); interestingly, this result was observed regardless of the fact whether the variants were analyzed individually or as a weighted genetic risk score [7]. A large meta-analysis of genome-wide association studies (GWAS) from the CHARGE consortium identified 3 genomic regions associated with common CIMT and 2 different regions associated with the presence of carotid plaque [8].

Several previous studies focused on the associations between genetic variants and atherosclerosis in RA patients. However, it should be underlined that most of these studies were performed by one group in a single (Spanish) population (Supplementary Table S1). Moreover, it must be emphasized that these studies used different parameters as a marker of atherosclerosis, i.e., CIMT, carotid plaque, CV events, flow-mediated endothelium-dependent vasodilatation FMD (postischemia), endothelium-independent vasodilatation NTG (postnitroglycerin), coronary artery calcification. In general, most of the genetic variants previously associated with CAD, CIMT or carotid plaque showed no association with atherosclerosis in RA patients.

In this study we aimed to evaluate the association between 29 previously reported SNPs and subclinical atherosclerosis in a cohort of Polish RA patients. We also sought to assess cumulative effects of the analyzed SNPs on subclinical atherosclerosis risk with the use of genetic risk scores.

\section{Methods}

The study was approved by the Military Institute of Medicine Ethics Committee (protocol number: 115/WIM/2007, date: 20.06.2007). Informed consent was obtained from each patient. All procedures were performed in accordance with the Helsinki Declaration of 1975, as revised in 1983.

\section{Control and study groups}

\section{Controls}

A group of 102 healthy individuals was used to determine normal values of CIMT in general population. Participants of the control group, 52 women and 50 men, were recruited among hospital staff. To ensure a representativity of the control group we enrolled 10 males and 10 females for each 10-year interval of age from 20 to 60 years and $\sim 10$ males and 10 females above 60 years. The exclusion criteria were: (1) the presence of chronic cardiovascular disease (except for well-controlled hypertension); (2) history of smoking;
(3) diabetes mellitus; (4) malignancy; (3) dyslipidaemia; and (6) renal insufficiency. The control group allowed for the construction of a statistically significant regression model for the relationship between age and CIMT in both men and women.

\section{Study group}

Two-hundred and eighty-nine consecutive RA patients (agreeing to participate in the study, fulfilling the 1987 ACR criteria and inclusion/exclusion criteria) were recruited from July 2007 to December 2012 in the Department of Internal Diseases and Rheumatology, Military Institute of Medicine, Warszawa, Poland. Exclusion criteria comprised: (1) clinically overt atherosclerotic disease (i.e., history of myocardial infarction, coronary revascularization or coronary artery by-pass graft, coronary artery disease confirmed by coronary angiography, myocardial scintigraphy or exercise stress test, history of stroke, clinical symptoms of lower extremity occlusive disease); (2) diabetes mellitus; (3) chronic kidney disease; (4) another coexisting autoinflammatory disease; and (5) history of malignancy. The study group was divided into 2 subgroups: (1) subclinical atherosclerosis group (SA) - patients with increased CIMT and/ or carotid plaque(s) and (2) non-subclinical atherosclerosis group (NSA) - patients without increased CIMT and without carotid plaque. Among 116 SA patients 73 patients had increased CIMT, 28 patients had increased CIMT and carotid plaque(s) and 15 patients had solely carotid plaque(s). Two sets of cardiovascular risk factors were used in the study: classical (age, sex, smoking, hypertension, body mass index-BMI, plasma lipids concentrations) and RA-related (rheumatoid factor-RF, anti-citrullinated protein antibodies-ACPA, disease duration).

\section{Ultrasonography}

Ultrasonography was performed with GE Logiq 5 Expert with a 7-12 MHz linear array transducer. Atherosclerotic plaques and IMT were assessed according to a protocol described elsewhere [9]. According to the recommendations of the American Society of Echocardiography CIMT greater than the 75th percentile for age and sex was classified as increased [10]. A group of 102 healthy individuals was used to determine normal values of CIMT in general population and to compute formulas for 75 th percentile.

\section{Single nucleotide polymorphisms (SNPs) selection}

SNPs associated with coronary artery disease, CIMT and carotid plaque from two large meta-analyses were selected for this study $[8,11]$. A SNP with minor allele frequency $(\mathrm{MAF})<0.05$ in European populations (i.e., rs3798220) 
was substituted by another $L P A$ SNP associated with CAD (i.e., rs10455872, MAF $=0.07$ in European populations). Two other SNPs (i.e., rs2306374 in the MRAS gene and rs599839 in the SORT1 gene) were substituted by their proxies: rs9818870 and rs646776, respectively. The full list of genotyped and analyzed SNPs is provided in Supplementary Table S2.

\section{Genotyping}

DNA was isolated from whole-blood samples using a salting-out method [12]. For SNPs genotyping a custom array was designed (Taqman OpenArray Genotyping Plate, Custom Format 16 QuantStudio 12K Flex, Life Technologies); genotyping was performed according to the manufacturer's protocols on a QuantStudio 12K Flex Real-Time PCR System (Applied Biosystems). All genotyped SNPs were in Hardy-Weinberg equilibrium. However, the $\mathrm{SH} 2 \mathrm{~B} 3$ gene SNP rs3184504 was excluded from the analysis because of the low genotyping success rate $(<90 \%)$. Finally, 29 SNPs were analyzed.

\section{Genetic risk scores (GRSs)}

The weighted genetic risk score was calculated according to the previously reported method [13] as $\Sigma w_{i} G_{i}$, where $G_{i}$ indicates the number of risk alleles $(0,1$ or 2$)$, while $\mathrm{w}_{\mathrm{i}}$ is the weight proportional to previously reported estimates of allelic effect (beta-coefficient or natural logarithm of the odds ratio). Three GRSs were calculated: (1) $\mathrm{GRS}_{\mathrm{IMT}}-\mathrm{GRS}$ comprising CIMT-associated SNPs; (2) GRS $\mathrm{CP}_{\mathrm{CP}}$-GR comprising carotid plaque-associated SNPs; and (3) $\mathrm{GRS}_{\mathrm{CAD}}$ GRS comprising CAD-associated SNPs. SNPs forming individual GRSs are listed in Supplementary Table S3. Because of the missing genotyping data, 16 subjects ( 9 SA and 7 NSA) were excluded from the GRS analysis.

\section{Statistical analysis}

The PLINK statistical software package was used to evaluate the differences in allele frequencies of each SNP between SA and NSA groups and to test the Hardy-Weinberg equilibrium (HWE) [14]. $P<0.05$ was considered as a significant deviation from the HWE. Other statistical analyses were performed using Statistica 12 package (StatSoft Inc). The Bonferroni correction (with a correction factor derived from the number of SNPs/GRSs tested) was used to adjust for multiple testing. Results are reported as mean (SD) for continuous variables and $n(\%)$ for categorical variables. According to data distribution, a parametric ( $\mathrm{t}$ test) or nonparametric ( $U$ Mann-Whitney) test were used. Categorical variables were compared with chi square exact test. GRSs in the SA and NSA groups were compared with the student's $t$ test. A logistic regression was used to predict the factors associated with subclinical atherosclerosis. A set of prediction models (comprising variables independently associated with subclinical atherosclerosis) was built. $P$ value $<0.05$ was considered significant.

\section{Results}

Subclinical atherosclerosis (SA) and non-subclinical atherosclerosis (NSA) groups comprised 116 and 173 patients, respectively. Groups characteristics are presented in Table 1. The SA and NSA patients differed in terms of age and smoking (60.3 years vs. 55.6 years, $P=0.002$, and 12.7 pack-years vs. 6.8 pack-years, $P=0.002$, respectively). The percentage of hypertensive patients was higher in SA patients, but the difference was not significant.

Out of 29 analyzed SNPs only 1 (rs4773144 in COL4A1) showed a marginal association with subclinical atherosclerosis (OR 1.43, 95\% CI 1.01-2.03, $P=0.049)$ (Supplementary Table S4); however, this association did not withstand the Bonferroni correction. Two SNPs showed a trend toward an association with subclinical atherosclerosis: rs6601530 in PINXI and rs445925 in APOCl.

The associations of the GRSs with subclinical atherosclerosis are presented in Table 2. Only GRS IMT $_{\text {IM }}$ showed a marginal association with subclinical atherosclerosis $(P=0.019$, $\left.P_{\text {corr }}=0.057\right)$.

A logistic regression analysis involving $\mathrm{GRS}_{\mathrm{IMT}}$, presence of RF and ACPA, and classical CV risk factors (i.e., age, sex, smoking, hypertension, BMI, plasma lipids concentrations) showed that only two parameters were independently associated with subclinical atherosclerosis: $\mathrm{GRS}_{\mathrm{IMT}}$ and age. A comparison of the 2 models, i.e., model 1 (comprising solely $\mathrm{GRS}_{\mathrm{IMT}}, P_{\text {model }}=0.02$ ) and model 2 (comprising GRS $_{\text {IMT }}$ and age $\left.P_{\text {model }}=0.0005\right)$ showed that adding age to GRS $_{\text {IMT }}$ increased the discriminative ability (OR 2.33 vs. 1.26 , accuracy $63 \%$ vs. $60.6 \%$ for model 1 and 2, respectively, $P_{\text {model } 1 \text { vs. } 2}=0.002$ ).

To assess the total risk conferred by $\mathrm{GRS}_{\mathrm{IMT}}$, we conducted a post-hoc analysis and calculated ORs according to GRS $_{\text {IMT }}$ tertile, using the first tertile as the reference group (Table 3). The subclinical atherosclerosis OR for top vs.

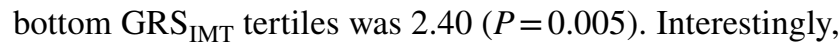
there were as many as 55 patients $(51.4 \%)$ in the highest tertile category.

Subsequently, we analyzed the influence of several factors (sex, RF, ACPA, hypertension, smoking, disease duration) on the association between GRS $_{\text {IMT }}$ and subclinical atherosclerosis (Tables 4, 5, Supplementary Table S5). These analyses showed that this association was limited to patients within first quartile of RA duration (Table 4): GRS $_{\text {IMT }} 0.025$ vs. 0.014 in SA and NSA group, respectively 
Table 1 SA and NSA group characteristics

\begin{tabular}{lccl}
\hline & SA $(n=116)$ & NSA $(n=173)$ & $P$ value \\
\hline Age, years & $60.3(12.3)$ & $55.6(12.8)$ & 0.002 \\
Males & $21(18.1 \%)$ & $28(16.2 \%)$ & 0.67 \\
Pack-years & $12.7(19.5)$ & $6.8(12.3)$ & 0.002 \\
BMI, kg/m ${ }^{2}$ & $25.1(4.1)^{\mathrm{a}}$ & $25.9(4.5)^{\mathrm{b}}$ & 0.17 \\
Hypertension & $55(47.4 \%)$ & $65(37.6 \%)$ & 0.096 \\
Total cholesterol, mg/dL & $203.9(39.2 .3)$ & $203.5(43.3)$ & 0.95 \\
LDL cholesterol, mg/dL & $116.5(32.5)^{\mathrm{c}}$ & $114.0(35.1)^{\mathrm{d}}$ & 0.54 \\
HDL cholesterol, mg/dL & $63.3(20.6)^{\mathrm{c}}$ & $63.7(19.9)^{\mathrm{d}}$ & 0.86 \\
Triglycerides, mg/dL & $127.1(56.7)^{\mathrm{c}}$ & $129.3(63.6)^{\mathrm{d}}$ & 0.77 \\
RF positivity & $82(71.3 \%)^{\mathrm{c}}$ & $115(69.3 \%)^{\mathrm{e}}$ & 0.72 \\
ACPA positivity & $78(77.2 \%)^{\mathrm{f}}$ & $116(76.9 \%)^{\mathrm{g}}$ & 0.94 \\
Current or previous DMARDs treatment & & & \\
Methotrexate & $102(87.9 \%)$ & $156(90.2 \%)$ & 0.43 \\
Sulfasalazine & $53(45.7 \%)$ & $87(50.3 \%)$ & 0.44 \\
Leflunomide & $45(38.8 \%)$ & $67(38.7)$ & 1.00 \\
Chloroquine/hydroxychloroquine & $36(31.0 \%)$ & $54(31.2 \%)$ & 1.00 \\
Cyclosporine A & $24(20.7 \%)$ & $47(27.2 \%)$ & 0.21 \\
Biologics & $19(16.3 \%)$ & $37(21.4 \%)$ & 0.29 \\
Number of DMARDs used & & & 0.8 \\
0 & $10(8.6 \%)$ & $14(8.1 \%)$ & \\
1 & $19(16.4 \%)$ & $24(13.9 \%)$ & \\
2 & $30(25.9 \%)$ & $52(30.1 \%)$ & \\
$\geq 3$ & $57(49.1 \%)$ & $83(48.0 \%)$ & \\
Current or previous glucocorticosteroids treatment & $110(94.8 \%)$ & $166(95.9 \%)$ & 1.00 \\
\hline & $12(10.4 \%)^{\mathrm{c}}$ & $17(10.2 \%)^{\mathrm{e}}$ & 1.00 \\
\hline
\end{tabular}

Data are presented as a mean (standard deviation) for continuous variables and as a number (percentage) for categorical variables

$A C P A$ anti-citrullinated protein antibodies, $B M I$ body mass index, GCS glucocorticosteroids, NSA non-subclinical atherosclerosis group, $R F$ rheumatoid factor, $S A$ subclinical atherosclerosis group

${ }^{a}$ Data available for 110 patients

${ }^{\mathrm{b}}$ Data available for 162 patients

${ }^{\mathrm{c}}$ Data available for 115 patients

${ }^{\mathrm{d}}$ Data available for 163 patients

${ }^{\mathrm{e}}$ Data available for 166 patients

${ }^{\mathrm{f}}$ Data available for 101 patients

${ }^{g}$ Data available for 151 patients

\begin{tabular}{llll}
\hline Genetic risk score & SA group $(n=107)$ & NSA group $(n=166)$ & $P$ \\
\hline GRS $_{\text {CAD }}$ & $1.152(0.153)$ & $1.134(0.163)$ & 0.34 \\
GRS $_{\text {IMT }}$ & $0.020(0.010)$ & $0.017(0.010)$ & 0.019 \\
GRS $_{\text {CP }}$ & $0.060(0.059)$ & $0.062(0.060)$ & $\left(P_{\text {corr }}=0.057\right)$ \\
\hline
\end{tabular}

Data are presented as a mean (SD)

$G R S$ genetic risk score, $G R S_{C A D}$ GRS comprising CAD-associated SNPs, $G R S_{I M T}$ GRS comprising IMTassociated SNPs, $G R S_{C P}$ GRS comprising carotid plaque-associated SNPs, NSA non-subclinical atherosclerosis group, $S A$ subclinical atherosclerosis group
$(P=0.00004)$ - the mean value of $\mathrm{GRS}_{\mathrm{IMT}}$ (i.e., mean value of GRS $_{\text {IMT }}$ for SA and NSA patients) was comparable across all quartiles (0.020 for Q1, 0.019 for Q2, 0.021 for $\mathrm{Q} 3$ and 0.020 for $\mathrm{Q} 4, P>0.05$ for all comparisons) but the 
Table 3 Distribution of SA and NSA cases in tertiles of GRS IMT $_{\text {T }}$

\begin{tabular}{lllll}
\hline Tertile of GRS & $\begin{array}{l}\text { No of } \\
\text { cases }\end{array}$ & & OR & $P$ \\
\cline { 2 - 3 } & NSA & SA & \\
\hline T1 $\left(\right.$ GRS $\left._{\text {IMT }} \leq 0.0078\right)$ & 55 & 21 & & \\
T2 $\left(0.0078<\right.$ GRS $\left._{\text {IMT }}<0.0234\right)$ & 51 & 31 & $1.59(0.81-3.12)$ & 0.17 \\
$\mathrm{~T}^{2}\left(\right.$ GRS $\left._{\text {IMT }} \geq 0.0234\right)$ & 60 & 55 & $2.40(1.29-4.47)$ & 0.005
\end{tabular}

$G R S_{I M T}$ GRS comprising carotid intima-media thickness-associated SNPs, NSA non-subclinical atherosclerosis group, $O R$ odds ratio, $S A$ subclinical atherosclerosis group

differential distribution of GRS IMT $_{\text {in SA and NSA groups }}$ was limited to Q1. The analysis of the relationship between the incidence of subclinical atherosclerosis and RA duration (Supplementary Figure S1) showed the highest incidence in early RA stages, then the incidence gradually decreased. The SA and NSA patients in the lowest disease duration quartile (Q1) did not differ significantly in terms of age $(P=0.2)$ and the association between GRS $_{\mathrm{IMT}}$ and subclinical atherosclerosis in these patients was present in both men $\left(\mathrm{GRS}_{\text {IMT }}\right.$ $0.0243 \pm 0.007$ vs. $0.0146 \pm 0.009, P=0.011)$ and woman
$\left(\mathrm{GRS}_{\mathrm{IMT}} 0.0242 \pm 0.012\right.$ vs. $\left.0.0158 \pm 0.010, P=0.002\right)$. The analysis of the differences between patients with short and long RA duration (i.e., Q1 vs. Q2-Q4) showed significant differences in treatment regimens. Q1 patients were more frequently DMARDs-naïve (15/68 vs. $\left.5 / 205, P=1.3 \times 10^{-6}\right)$ and the percentage of patients treated with biologics was significantly lower ( $8 / 68$ vs. $48 / 204, P=0.038)$. Further analysis revealed that the association between GRS IMT $_{\text {and sub- }}$ clinical atherosclerosis is limited to an even shorter period of disease duration (Table 4)-there were no difference in GRS $_{\text {IMT }}$ between SA and NSA groups in patients with RA duration $>2$ years $(P=0.44)$ and $>1$ year $(P=0.22)$, while the difference was significant in patients with disease duration $\leq 2$ years $(P=0.0002)$ or $\leq 1$ year $(P=0.002)$. In addition, GRS $_{\text {IMT }}$ was significantly higher in SA patients with RA duration $\leq 2$ years and $\leq 1$ year as compared to SA patients with RA duration $>2$ years and $>1$ year, respectively (Table 5). Almost one-third of patients with RA duration $\leq 2$ years were DMARDs-naïve, while in patients with RA duration $\leq 1$ year, this percentage was as high as 48.2 (Table 5). Patients with RA duration $\leq 1$ year and $\leq 2$ years did not differ significantly from patients with RA duration $>1$ year and $>2$ years in terms of age $(55.9 \pm 13.3$ years

Table 4 Relation between GRS $\mathrm{IMT}_{\text {IM }}$ and subclinical atherosclerosis according to rheumatoid arthritis duration

\begin{tabular}{|c|c|c|c|c|}
\hline & \multirow[t]{2}{*}{ RAD } & \multicolumn{2}{|l|}{$\mathrm{GRS}_{\mathrm{IMT}}$} & \multirow[t]{2}{*}{$P$} \\
\hline & & SA & NSA & \\
\hline \multirow[t]{4}{*}{ Quartile of RAD } & Q1 (RAD < 60 months) & $0.025(0.011), n=27$ & $0.014(0.010), n=41$ & 0.00004 \\
\hline & Q2 (60 months $\leq$ RAD $<108$ months) & $0.017(0.011), n=29$ & $0.016(0.010), n=36$ & 0.71 \\
\hline & Q3 (108 months $\leq$ RAD $<204$ months) & $0.018(0.011), n=27$ & $0.020(0.011), n=41$ & 0.64 \\
\hline & Q4 (RAD $\geq 204$ months) & $0.019(0.008), n=21$ & $0.017(0.011), n=44$ & 0.51 \\
\hline \multirow[t]{2}{*}{ RAD $\leq 1$ year vs. $>1$ year } & $\leq 1$ year & $0.029(0.013), n=12$ & $0.015(0.009), n=15$ & 0.002 \\
\hline & $>1$ year & $0.019(0.011), n=95$ & $0.017(0.01), n=151$ & 0.22 \\
\hline \multirow[t]{2}{*}{$\mathrm{RAD} \leq 2$ years vs. $>2$ years } & $\leq 2$ years & $0.027(0.011), n=19$ & $0.014(0.01), n=27$ & 0.0002 \\
\hline & $>2$ years & $0.019(0.010), n=88$ & $0.017(0.01), n=139$ & 0.44 \\
\hline
\end{tabular}

Data are presented as mean (SD)

$G R S_{I M T}$ GRS comprising carotid intima-media thickness-associated SNPs, NSA non-subclinical atherosclerosis group, $Q 1-Q 4$ quartiles of rheumatoid arthritis duration, $R A D$ rheumatoid arthritis duration, $S A$ subclinical atherosclerosis group

Table 5 Relation between GRS $_{\mathrm{IMT}}$ and subclinical atherosclerosis, and the differences in DMARDs treatment according to rheumatoid arthritis duration

\begin{tabular}{lll}
\hline & RAD $\leq 1$ year $(n=27)$ vs. $>1$ year $(n=246)$ & RAD $\leq 2$ years $(n=46)$ vs. $>2$ years $(n=227)$ \\
\hline GRS $_{\mathrm{IMT}}$ in SA patients & $0.029(0.013)$ vs. $0.019(0.010), P=9 \times 10^{-4}$ & $0.027(0.011)$ vs. $0.019(0.010), P=0.0018$ \\
GRS $_{\mathrm{IMT}}$ in NSA patients & $0.015(0.009)$ vs. $0.017(0.010), P=0.36$ & $0.014(0.010)$ vs. $0.017(0.010), P=0.13$ \\
DMARDs-naïve patients, $n(\%)$ & $13(48.2 \%)$ vs. $7(2.8 \%), P=1.9 \times 10^{-10}$ & $14(30.4 \%)$ vs. $6(2.6 \%), P=4.2 \times 10^{-8}$ \\
Patients on biologics, $n(\%)$ & $3(11.1 \%)$ vs. $53(21.6 \%), P=0.2$ & $5(10.9 \%)$ vs. $51(22.6 \%), P=0.08$ \\
\hline
\end{tabular}

Data are presented as mean (SD) or number (\%)

$D M A R D s$ disease modifying anti-rheumatic drugs, $G R S_{I M T}$ GRS comprising carotid intima-media thickness-associated SNPs, NSA non-subclinical atherosclerosis group, $R A D$ rheumatoid arthritis duration, $S A$ subclinical atherosclerosis group 
vs. $57.6 \pm 12.9$ years, $P=0.46$ and $55.1 \pm 12.5$ years vs. $57.9 \pm 12.9$ years, $P=0.17$ for RA duration $\leq 1$ year vs. $>1$ year and $\leq 2$ years vs. $>2$ years, respectively).

The analysis of other subgroups (smoking, RF, ACPA, hypertension) showed no significant associations (only marginal association was found in ACPA-positive patients) (Supplementary Table S5).

\section{Discussion}

The pathogenesis of atherosclerosis is complex, since it involves genetic predisposition as well as environmental factors. Several genetic loci were found to be associated with atherosclerosis; however, the influence of a single locus is modest. A GRS, combining several genetic variants, is a tool to assess cumulative effects of genetic factors on disease risk. Several previous studies showed usefulness of GRSs in the prediction of cardiovascular disease [15-18].

The associations of CAD risk variants SNPs with subclinical atherosclerosis were tested in several studies. Studies by Conde et al. and by Zabalza et al. did not find an association between CAD risk variants and subclinical atherosclerosis $[6,7]$. One might speculate that the effects of a single variant might be very modest; if this is the case, a GRS might allow finding an association. However, the studies evaluating associations of GRSs based on clinical atherosclerosis risk variants gave discrepant results. On one hand, Hamrefors et al. found that a myocardial infarction GRS was associated with markers of carotid atherosclerosis (i.e., CIMT and plaque) [19], and Salfati et al. showed that a GRS for clinical CAD was associated with advanced coronary atherosclerosis [20]. On the other hand, a large-scale study performed in three Finnish cohorts showed that none of the 24 genomewide significant coronary artery disease risk variants was associated with subclinical atherosclerosis and a GRS combining those variants did not improve the prediction of subclinical atherosclerosis [21]. Similar results were showed by Zabalza et al._-a GRS comprising 9 CAD risk variants was not associated with subclinical atherosclerosis [7]. Thus, it is possible that subclinical atherosclerosis is, at least partially, driven by other genetic factors than those involved in clinically overt atherosclerosis. This hypothesis is strongly supported by the large meta-analysis by Bis et al. (over 40,000 participants of European ancestry), which demonstrated that none of the SNPs reported to be associated with coronary artery disease in the CARDIoGRAM consortium had a significant association with CIMT or carotid plaque [8]. Moreover, the study identified 3 genetic variants associated with CIMT and 2 associated with carotid plaque (and further 3 SNPs with suggestive evidence for association with CIMT and carotid plaque); interestingly, most of these loci showed no association with CAD [8].
Several previous studies evaluated associations between $\mathrm{CAD} /$ subclinical atherosclerosis risk variants and atherosclerosis in RA patients (most studies performed in Spanish population), but most of them gave negative results (Supplementary Table S1). In addition, a large-scale study involving 91 CAD genetic variants showed an association of only few of them with subclinical atherosclerosis (defined as CAC) in RA patients and the authors suggested that the increased risk of CAC in RA did not appear to operate primarily through established genetically regulated atherogenic mechanisms that are preponderant in general population [22].

We investigated an association between $29 \mathrm{CAD} /$ subclinical atherosclerosis-associated SNPs and subclinical atherosclerosis in a Polish RA cohort. Of these 29 SNPs, only 1 (rs4773144 in COLAA1) showed a marginal association with subclinical atherosclerosis and two SNPs showed a trend toward association: rs6601530 in PINX1 and rs445925 in APOC1. Interestingly, 2 of the above-mentioned SNPs were previously associated with CIMT [8].

We also investigated the associations of 3 GRSs with subclinical atherosclerosis. However, only GRS $_{\text {IMT }}$ (combining 3 SNPs previously associated with CIMT in a large metaanalysis by Bis et al. [8]) showed a marginal association with subclinical atherosclerosis. The analysis of distribution of SA and NSA cases according to the GRS $_{\text {IMT }}$ tertile showed a significant increase in subclinical atherosclerosis risk in the top tertile as compared to the bottom tertile (OR 2.40, $P=0.005)$. Our results suggest that in RA, similarly to general population, subclinical atherosclerosis may be driven by different genetic factors than those present in clinically overt atherosclerosis.

A logistic regression showed that $\mathrm{GRS}_{\mathrm{IMT}}$ and age were independently associated with subclinical atherosclerosis. Lack of association with BMI and lipids may be explained by RA pathophysiology. It is well known that active RA may lead to weight loss and abnormal body composition (decreased lean muscle mass and increased adiposity). Moreover, most studies reported a higher CV mortality in RA patients with a low body mass index (BMI) $(<20 \mathrm{~kg} /$ $\left.\mathrm{m}^{2}\right)$ as compared to patients with a higher BMI $\left(\geq 30 \mathrm{~kg} / \mathrm{m}^{2}\right)$ [23]. Thus, in RA abnormal body composition (increased adiposity) may be a better predictor of CVD than BMI. As for lipids, RA is associated with altered lipid profile-the so-called lipid paradox, where in spite of lower total cholesterol, low-density lipoprotein (LDL) cholesterol and high-density lipoprotein (HDL) cholesterol patients remain at high risk of CVD developing [24]. Moreover, quantitative changes in lipid profile are accompanied by functional alterations, such as impaired HDLc efflux capacity (the ability to move cholesterol from lipid laden macrophages) [24]. The sex distribution was similar in SA and NSA groups. As for hypertension, we did not demonstrate its association with subclinical atherosclerosis, however, a trend towards some 
association was observed. Smoking was associated with subclinical atherosclerosis in univariate (but not in multivariate) analysis. As the role of smoking, male sex and hypertension is well established in atherosclerosis, it is obvious that all these factors contribute to its development; lack of association in our study was rather due to a small study group. On the other hand, it might also indicate that the relative contribution of these factors to atherosclerosis is smaller as compared to age or genetic factors.

We also investigated if combining classical and genetic risk factors improved the prediction of subclinical atherosclerosis. The results demonstrated that adding age to GRS $_{\text {IMT }}$ significantly improved the predictive accuracy of the model. Thus, our study suggests that in RA, similarly to general population, the combination of genetic and clinical risk factors may improve the prediction of atherosclerosis $[15,16]$.

Our results, at first glance, seem to suggest that in Polish RA population subclinical atherosclerosis may be driven by similar genetic factors as in general population (association of GRS $_{\text {IMT }}$ with subclinical atherosclerosis). Thus, our results would contrast with most previous studies. However, subgroup analysis showed an interesting finding: the association between GRS $_{\text {IMT }}$ and subclinical atherosclerosis was limited to patients with shortest RA duration. One may speculate that in early rheumatoid arthritis, the contribution of common (i.e., present in general population) genetic and non-genetic factors to subclinical atherosclerosis is large; while in long-lasting RA, the role of disease-related factors gradually increases, thus, the relative contribution of common risk factors (including common genetic risk factors) decreases. Another possible explanation for our observation is the difference in treatment regimens. Previous studies showed that subclinical atherosclerosis may accelerate early during the disease course or even in the preclinical state [25]. It is well established that effective treatment with disease modifying anti-rheumatic drugs (DMARDs) may abrogate the effect of RA on atherosclerosis progression. However, it should be emphasized that in a large proportion of patients a substantial delay from symptom onset to RA diagnosis exists-epidemiological studies showed that in Poland the median delay from symptom onset to assessment by the rheumatologist was 35 weeks [26]. Moreover, conventional DMARDs (first-line RA therapy) are slowacting drugs, so during the first months of treatment their anti-inflammatory effect is limited. A study by Raczkiewicz et al., involving patients with early RA treated according to current recommendations, showed a rapid progression of CIMT in the first year of treatment, which slowed down in the second year [27]. Interestingly, some studies showed that DMARDs may not only slow down the increase of CIMT but even cause its reduction [28, 29]. Thus, it seems that DMARDs may have a strong effect on atherosclerosis, influencing not only RA-related but also classical CV risk factors. This hypothesis is supported by several studies performed in non-RA population which proved a beneficial effect of anti-inflammatory treatment on atherosclerosis. The LoDoCo trial showed that low-dose colchicine prevented recurrent cardiovascular events in patients with stable CAD [30]. The CANTOS study showed similar results for canakinumab [31]. In addition, the ongoing Cardiovascular Inflammation Reduction Trial (CIRT) tests the influence of low-dose methotrexate (anchor drug for RA) on cardiovascular risk $[32,33]$. Our results seem to support those mentioned above. We observed a high incidence of subclinical atherosclerosis in early stages of RA, which, subsequently, gradually decreased. This high incidence in early stages of RA may be due to the considerable proportion of DMARDsnaïve patients, as well as to the fact that in some patients DMARDs might have not reach their maximal effect yet. If this is the case, our results should not be considered as discrepant from previous studies, but as those which highlight the role of RA as an independent cardiovascular risk factor and the beneficial effect of DMARDs on the CV risk. In this context, it would be very interesting to re-analyze previous studies taking into account RA duration and treatment regimens.

A possible modifying effect of DMARDs on genetic risk of atherosclerosis is not easy to explain. However, it should be emphasized that many genes associated with atherosclerosis encode proteins involved in inflammatory processes and thus, the effect of their changed expression might be influenced by immunosuppressive/immunomodulatory drugs, e.g., methotrexate. Such a gene (i.e., involved in inflammatory processes) is $A P O C l-a$ component of $\mathrm{GRS}_{\text {IMT }}$, which was shown not only to be associated with lipids but also with systemic inflammation [34].

We acknowledge some limitations to this study. First, the study group was relatively small, and therefore, our study had limited power to detect associations of single SNPs with subclinical atherosclerosis. To overcome this problem, we used a weighted GRS as the main genetic variable. Second, we did not have a replication cohort. Thus, our results need to be confirmed by other studies.

In conclusion, we found that GRS IMT $_{\text {I }}$ was associated with subclinical atherosclerosis in RA patients; however, the association was limited to patients with short RA duration. In this subgroup of RA patients, subclinical atherosclerosis seemed to be, at least partially, driven by similar genetic factors as in general population. In contrast, in patients with long-lasting RA, the role of common genetic factors seemed to be relatively small. Further studies are needed to elucidate the relationship between common genetic risk factors and atherosclerosis in RA. However, our study suggests that future studies should take into account disease duration and RA-specific treatment. 
Author contributions BK: conception and design of the work; acquisition, analysis and interpretation of data for the work; drafting the manuscript; final approval of the version to be published; agreement to be accountable for all aspects of the work in ensuring that questions related to the accuracy or integrity of any part of the work are appropriately investigated and resolved. RK: acquisition of data for the work; revising the manuscript critically for important intellectual content; final approval of the version to be published; agreement to be accountable for all aspects of the work in ensuring that questions related to the accuracy or integrity of any part of the work are appropriately investigated and resolved. AJ: acquisition of data for the work; revising the manuscript critically for important intellectual content; final approval of the version to be published; agreement to be accountable for all aspects of the work in ensuring that questions related to the accuracy or integrity of any part of the work are appropriately investigated and resolved. AR: acquisition of data for the work; revising the manuscript critically for important intellectual content; final approval of the version to be published; agreement to be accountable for all aspects of the work in ensuring that questions related to the accuracy or integrity of any part of the work are appropriately investigated and resolved. $\mathrm{AB}$ : acquisition, analysis and interpretation of data for the work; revising the manuscript critically for important intellectual content; final approval of the version to be published; agreement to be accountable for all aspects of the work in ensuring that questions related to the accuracy or integrity of any part of the work are appropriately investigated and resolved. KK: acquisition of data for the work; revising the manuscript critically for important intellectual content; final approval of the version to be published; agreement to be accountable for all aspects of the work in ensuring that questions related to the accuracy or integrity of any part of the work are appropriately investigated and resolved. KD: acquisition of data for the work; revising the manuscript critically for important intellectual content; final approval of the version to be published; agreement to be accountable for all aspects of the work in ensuring that questions related to the accuracy or integrity of any part of the work are appropriately investigated and resolved. AM: design of the work; revising the manuscript critically for important intellectual content; final approval of the version to be published; agreement to be accountable for all aspects of the work in ensuring that questions related to the accuracy or integrity of any part of the work are appropriately investigated and resolved. KS: acquisition, analysis and interpretation of data for the work; revising the manuscript critically for important intellectual content; final approval of the version to be published; agreement to be accountable for all aspects of the work in ensuring that questions related to the accuracy or integrity of any part of the work are appropriately investigated and resolved. RP: conception and design of the work; analysis and interpretation of data for the work; revising the manuscript critically for important intellectual content; agreement to be accountable for all aspects of the work in ensuring that questions related to the accuracy or integrity of any part of the work are appropriately investigated and resolved. MS: analysis and interpretation of data for the work; revising the manuscript critically for important intellectual content; final approval of the version to be published; agreement to be accountable for all aspects of the work in ensuring that questions related to the accuracy or integrity of any part of the work are appropriately investigated and resolved. SN: analysis and interpretation of data for the work; revising the manuscript critically for important intellectual content; final approval of the version to be published; agreement to be accountable for all aspects of the work in ensuring that questions related to the accuracy or integrity of any part of the work are appropriately investigated and resolved. KK: analysis and interpretation of data for the work; revising the manuscript critically for important intellectual content; final approval of the version to be published; agreement to be accountable for all aspects of the work in ensuring that questions related to the accuracy or integrity of any part of the work are appropriately investigated and resolved. MT: acquisition, analysis and interpretation of data for the work; revising the manuscript critically for important intellectual content; final approval of the version to be published; agreement to be accountable for all aspects of the work in ensuring that questions related to the accuracy or integrity of any part of the work are appropriately investigated and resolved. WT: conception and design of the work; analysis and interpretation of data for the work; revising the manuscript critically for important intellectual content; agreement to be accountable for all aspects of the work in ensuring that questions related to the accuracy or integrity of any part of the work are appropriately investigated and resolved.

Funding This work was supported by Polish Ministry of Science and Higher Education Grant no. N N402 077234 (granted to Witold Tłustochowicz).

Data availability All data generated or analyzed during this study are included in this published article and its Supplementary Information files.

\section{Compliance with ethical standards}

Conflict of interest The authors declare that there is no conflict of interests regarding the publication of this paper.

Open Access This article is distributed under the terms of the Creative Commons Attribution 4.0 International License (http://creativeco mmons.org/licenses/by/4.0/), which permits unrestricted use, distribution, and reproduction in any medium, provided you give appropriate credit to the original author(s) and the source, provide a link to the Creative Commons license, and indicate if changes were made.

\section{References}

1. Aviña-Zubieta JA, Choi HK, Sadatsafavi M, Etminan M, Esdaile JM, Lacaille D (2008) Risk of cardiovascular mortality in patients with rheumatoid arthritis: a meta-analysis of observational studies. Arthritis Rheum 59:1690-1697. https://doi.org/10.1002/ art. 24092

2. del Rincon ID, Williams K, Stern MP, Freeman GL, Escalante A (2001) High incidence of cardiovascular events in rheumatoid arthritis cohort not explained by traditional cardiac risk factors. Arthritis Rheum 44:2737-2745

3. Lorenz MW, Markus HS, Bots ML, Rosvall M, Sitzer M (2007) Prediction of clinical cardiovascular events with carotid intimamedia thickness: a systematic review and meta-analysis. Circulation 115:459-467

4. Tyrrell PN, Beyene J, Feldman BM, McCrindle BW, Silverman ED, Bradley TJ (2010) Rheumatic disease and carotid intimamedia thickness: a systematic review and meta-analysis. Arterioscler Thromb Vasc Biol 30:1014-1026. https://doi.org/10.1161/ ATVBAHA.109.198424

5. Hartiala J, Schwartzman WS, Gabbay J, Ghazalpour A, Bennett BJ, Allayee H (2017) The genetic architecture of coronary artery disease: current knowledge and future opportunities. Curr Atheroscler Rep 19:6. https://doi.org/10.1007/s11883-017-0641-6

6. Conde L, Bevan S, Sitzer M, Klopp N, Illig T, Thiery J, Seissler J, Baumert J, Raitakari O, Kähönen M, Lyytikäinen LP, Laaksonen R, Viikari J, Lehtimäki T, Koernig W, Halperin E, Markus HS (2011) Novel associations for coronary artery disease derived from genome wide association studies are not associated with increased carotid intima-media thickness, suggesting they do not act via early atherosclerosis or vessel remodeling. 
Atherosclerosis 219:684-689. https://doi.org/10.1016/j.atheroscle rosis.2011.08.031

7. Zabalza M, Subirana I, Lluis-Ganella C, Sayols-Baixeras S, de Groot E, Arnold R, Cenarro A, Ramos R, Marrugat J, Elosua R (2015) Association between coronary artery disease genetic variants and subclinical atherosclerosis: an association study and meta-analysis. Rev Esp Cardiol (Engl Ed) 68:869-877. https://doi. org/10.1016/j.rec.2014.10.023

8. Bis JC, CARDIoGRAM Consortium et al (2011) Meta-analysis of genome-wide association studies from the CHARGE consortium identifies common variants associated with carotid intima media thickness and plaque. Nat Genet 43:940-947. https://doi. org/10.1038/ng.920

9. Kisiel B, Kruszewski R, Juszkiewicz A, Raczkiewicz A, Bachta A, Tłustochowicz M, Staniszewska-Varga J, Kłos K, Duda K, Bogusławska-Walecka R, Płoski R, Tłustochowicz W (2015) Methotrexate, cyclosporine A, and biologics protect against atherosclerosis in rheumatoid arthritis. J Immunol Res 2015:759610. https://doi.org/10.1155/2015/759610

10. Stein JH, Korcarz CE, Hurst RT, Lonn E, Kendall CB, Mohler ER, Najjar SS, Rembold CM, Post WS, American Society of Echocardiography Carotid Intima-Media Thickness Task Force (2008) Use of carotid ultrasound to identify subclinical vascular disease and evaluate cardiovascular disease risk: a consensus statement from the American Society of Echocardiography Carotid IntimaMedia Thickness Task Force. Endorsed by the Society for Vascular Medicine. J Am Soc Echocardiogr 21:93-111. https://doi. org/10.1016/j.echo.2007.11.011

11. Schunkert H, CARDIoGRAM Consortium et al (2011) Largescale association analysis identifies 13 new susceptibility loci for coronary artery disease. Nat Genet 43:333-338. https://doi. org/10.1038/ng.784

12. Miller SA, Dykes DD, Polesky HF (1988) A simple salting out procedure for extracting DNA from human nucleated cells. Nucleic Acids Res 16:1215

13. Zhang G, Karns R, Sun G, Indugula SR, Cheng H, HavasAugustin D, Novokmet N, Rudan D, Durakovic Z, Missoni S, Chakraborty R, Rudan P, Deka R (2011) Extent of height variability explained by known height-associated genetic variants in an isolated population of the Adriatic coast of Croatia. PLoS One 6:e29475. https://doi.org/10.1371/journal.pone.0029475

14. Purcell S, Neale B, Todd-Brown K, Thomas L, Ferreira MA, Bender D, Maller J, Sklar P, de Bakker PI, Daly MJ, Sham PC (2007) PLINK: a tool set for whole genome association and population-based linkage analyses. Am J Hum Genet 81:559-575

15. Lluis-Ganella C, Subirana I, Lucas G, Tomás M, Muñoz D, Sentí M, Salas E, Sala J, Ramos R, Ordovas JM, Marrugat J, Elosua R (2012) Assessment of the value of a genetic risk score in improving the estimation of coronary risk. Atherosclerosis 222:456-463. https://doi.org/10.1016/j.atherosclerosis.2012.03.024

16. Morrison AC, Bare LA, Chambless LE, Ellis SG, Malloy M, Kane JP, Pankow JS, Devlin JJ, Willerson JT, Boerwinkle E (2007) Prediction of coronary heart disease risk using a genetic risk score: the Atherosclerosis Risk in Communities Study. Am J Epidemiol 166:28-35

17. Krarup NT, Borglykke A, Allin KH, Sandholt CH, Justesen JM, Andersson EA, Grarup N, Jørgensen T, Pedersen O, Hansen T (2015) A genetic risk score of 45 coronary artery disease risk variants associates with increased risk of myocardial infarction in 6041 Danish individuals. Atherosclerosis 240:305-310. https ://doi.org/10.1016/j.atherosclerosis.2015.03.022

18. Brautbar A, Pompeii LA, Dehghan A, Ngwa JS, Nambi V, Virani SS, Rivadeneira F, Uitterlinden AG, Hofman A, Witteman JC, Pencina MJ, Folsom AR, Cupples LA, Ballantyne CM, Boerwinkle E (2012) A genetic risk score based on direct associations with coronary heart disease improves coronary heart disease risk prediction in the Atherosclerosis Risk in Communities (ARIC), but not in the Rotterdam and Framingham Offspring, Studies. Atherosclerosis 223:421-426. https://doi.org/10.1016/j.atheroscle rosis.2012.05.035

19. Hamrefors V, Hedblad B, Engström G, Almgren P, Sjögren M, Melander O (2012) A myocardial infarction genetic risk score is associated with markers of carotid atherosclerosis. J Intern Med 271:271-281. https://doi.org/10.1111/j.1365-2796.2011.02472.x

20. Salfati E, Nandkeolyar S, Fortmann SP, Sidney S, Hlatky MA, Quertermous T, Go AS, Iribarren C, Herrington DM, Goldstein BA, Assimes TL (2015) Susceptibility loci for clinical coronary artery disease and subclinical coronary atherosclerosis throughout the life-course. Circ Cardiovasc Genet 8:803-811. https://doi. org/10.1161/CIRCGENETICS.114.001071

21. Hernesniemi JA, Seppälä I, Lyytikäinen LP, Mononen N, Oksala N, Hutri-Kähönen N, Juonala M, Taittonen L, Smith EN, Schork NJ, Chen W, Srinivasan SR, Berenson GS, Murray SS, Laitinen T, Jula A, Kettunen J, Ripatti S, Laaksonen R, Viikari J, Kähönen M, Raitakari OT, Lehtimäki T (2012) Genetic profiling using genome-wide significant coronary artery disease risk variants does not improve the prediction of subclinical atherosclerosis: the Cardiovascular Risk in Young Finns Study, the Bogalusa Heart Study and the Health 2000 Survey-a meta-analysis of three independent studies. PLoS One 7:e28931. https://doi.org/10.1371/ journal.pone.0028931

22. Ferraz-Amaro I, Winchester R, Gregersen PK, Reynolds RJ, Wasko MC, Oeser A, Chung CP, Stein CM, Giles JT, Bathon JM (2017) Coronary artery calcification and rheumatoid arthritis: lack of relationship to risk alleles for coronary artery disease in the general population. Arthritis Rheumatol 69:529-541. https://doi. org/10.1002/art.39862

23. van den Oever IA, van Sijl AM, Nurmohamed MT (2013) Management of cardiovascular risk in patients with rheumatoid arthritis: evidence and expert opinion. Ther Adv Musculoskelet Dis 5:166-181. https://doi.org/10.1177/1759720X13491025

24. McGrath CM, Young SP (2015) Lipid and metabolic changes in rheumatoid arthritis. Curr Rheumatol Rep 17:57. https://doi. org/10.1007/s11926-015-0534-Z

25. Kerola AM, Kauppi MJ, Kerola T, Nieminen TV (2012) How early in the course of rheumatoid arthritis does the excess cardiovascular risk appear? Ann Rheum Dis 71:1606-1615. https://doi. org/10.1136/annrheumdis-2012-201334

26. Raza K, Stack R, Kumar K, Filer A, Detert J, Bastian H, Burmester GR, Sidiropoulos P, Kteniadaki E, Repa A, Saxne T, Turesson C, Mann H, Vencovsky J, Catrina A, Chatzidionysiou A, Hensvold A, Rantapää-Dahlqvist S, Binder A, Machold K, Kwiatkowska B, Ciurea A, Tamborrini G, Kyburz D, Buckley CD (2011) Delays in assessment of patients with rheumatoid arthritis: variations across Europe. Ann Rheum Dis 70:1822-1825. https:// doi.org/10.1136/ard.2011.151902

27. Raczkiewicz A, Juszkiewicz A, Kisiel B, Bachta A, Kur-Zalewska J, Kłos K, Bujakowska O, Tłustochowicz M, Tłustochowicz W (2016) Treat-to-target therapy does not prevent excessive progression of carotid intima media thickness during the first year of therapy in early rheumatoid arthritis. Arch Med Sci Atheroscler Dis 1:e36-e43. https://doi.org/10.5114/amsad.2016.60225

28. Novikova DS, Popkova TV, Lukina GV, Luchikhina EL, Karateev DE, Volkov AV, Novikov AA, Aleksandrova EN, Nasonov EL (2016) The effects of rituximab on lipids, arterial stiffness and carotid intima-media thickness in rheumatoid arthritis. J Korean Med Sci 31:202-207. https://doi.org/10.3346/jkms.2016.31.2.202

29. Angel K, Provan SA, Fagerhol MK, Mowinckel P, Kvien TK, Atar D (2012) Effect of 1-year anti-TNF- $\alpha$ therapy on aortic stiffness, carotid atherosclerosis, and calprotectin in inflammatory arthropathies: a controlled study. Am J Hypertens 25:644-650. https://doi. org/10.1038/ajh.2012.12 
30. Nidorf SM, Eikelboom JW, Budgeon CA, Thompson PL (2013) Low-dose colchicine for secondary prevention of cardiovascular disease. J Am Coll Cardiol 61:404-410. https://doi.org/10.1016/j. jacc.2012.10.027

31. Ridker PM, Everett BM, Thuren T, MacFadyen JG, Chang WH, Ballantyne C, Fonseca F, Nicolau J, Koenig W, Anker SD, Kastelein JJP, Cornel JH, Pais P, Pella D, Genest J, Cifkova R, Lorenzatti A, Forster T, Kobalava Z, Vida-Simiti L, Flather M, Shimokawa H, Ogawa H, Dellborg M, Rossi PRF, Troquay RPT, Libby P, Glynn RJ, CANTOS Trial Group (2017) Antiinflammatory therapy with canakinumab for atherosclerotic disease. N Engl J Med 377:1119-1131. https://doi.org/10.1056/NEJMoa1707914

32. Ridker PM (2009) Testing the inflammatory hypothesis of atherothrombosis: scientific rationale for the cardiovascular inflammation reductiontrial (CIRT). J Thromb Haemost 7:332339. https://doi.org/10.1111/j.1538-7836.2009.03404.x

33. Everett BM, Pradhan AD, Solomon DH, Paynter N, Macfadyen J, Zaharris E, Gupta M, Clearfield M, Libby P, Hasan AA, Glynn RJ, Ridker PM (2013) Rationale and design of the Cardiovascular Inflammation Reduction Trial: a test of the inflammatory hypothesis of atherothrombosis. Am Heart J 166:199-207.e15. https:// doi.org/10.1016/j.ahj.2013.03.018

34. Ligthart S, de Vries PS, Uitterlinden AG, Hofman A, Franco $\mathrm{OH}$, Chasman DI, Dehghan A, CHARGE Inflammation Working Group (2015) Pleiotropy among common genetic loci identified for cardiometabolic disorders and C-reactive protein. PLoS One 10:e0118859. https://doi.org/10.1371/journal.pone.0118859

\section{Affiliations}

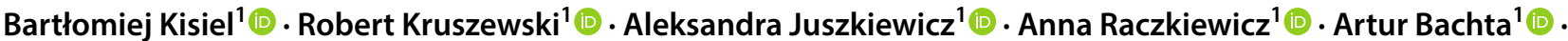

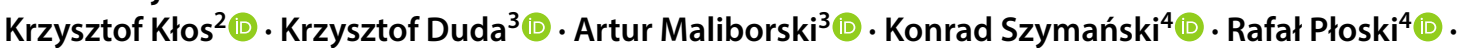

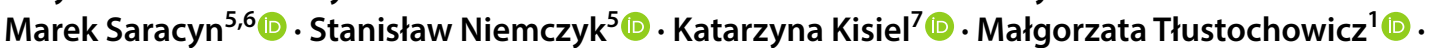 Witold Tłustochowicz ${ }^{1}$ [D}

Robert Kruszewski

rkruszewski@wim.mil.pl

Aleksandra Juszkiewicz

ajuszkiewicz@wim.mil.pl

Anna Raczkiewicz

araczkiewicz@wim.mil.pl

Artur Bachta

artur.bachta@gmail.com

Krzysztof Kłos

kklos@wim.mil.pl

Krzysztof Duda

kduda@wim.mil.pl

Artur Maliborski

artur.maliborski@gmail.com

Konrad Szymański

kszyma@gmail.com

Rafał Płoski

rploski@wp.pl

Marek Saracyn

msaracyn@interia.pl

Stanisław Niemczyk

sniemczyk@wim.mil.pl

Katarzyna Kisiel

kkilian@wp.pl
Małgorzata Tłustochowicz

mtlustochowicz@wim.mil.pl

Witold Tłustochowicz

wtlustochowicz@wim.mil.pl

1 Department of Internal Diseases and Rheumatology, Military Institute of Medicine, ul. Szaserów 128,

04-141 Warszawa, Poland

2 Department of Infectious Diseases and Allergology, Military Institute of Medicine, ul. Szaserów 128, 04-141 Warszawa, Poland

3 Department of Radiology, Military Institute of Medicine, ul. Szaserów 128, 04-141 Warszawa, Poland

4 Department of Medical Genetics, Medical University of Warsaw, ul. Pawińskiego 3c, 02-106 Warszawa, Poland

5 Department of Internal Diseases, Nephrology and Dialysis, Military Institute of Medicine, ul. Szaserów 128, 04-141 Warszawa, Poland

6 Department of Endocrinology and Isotope Therapy, Military Institute of Medicine, ul. Szaserów 128, 04-141 Warszawa, Poland

7 Department of Pediatric Dermatology, Center of Dermatology, Międzyleski Specialist Hospital, ul. Bursztynowa 2, 04-749 Warszawa, Poland 